\title{
A SIMPLISTIC APPROACH TO INVERSE TRANSVERSALS*
}

\author{
by T. S. BLYTH and M. H. ALMEIDA SANTOS
}

(Received 19th May 1994)

\begin{abstract}
An inverse transversal of a regular semigroup $S$ is an inverse subsemigroup that contains precisely one inverse of each element of $S$. In the literature there are three known types of inverse transversal, namely those that are multiplicative, those that are weakly multiplicative, and those that form quasi-ideals. Here, by considering natural ways in which certain words can be simplified, we reveal four new types of inverse transversal. All of these can be illustrated nicely in examples that are based on $2 \times 2$ matrices.
\end{abstract}

1991 Mathematics subject classification: 20M17.

An inverse transversal of a regular semigroup $S$ is an inverse subsemigroup $T$ with the property that $|T \cap V(x)|=1$ for every $x \in S$, where $V(x)$ denotes the set of inverses of $x \in S$. In what follows we shall write the unique element of $T \cap V(x)$ as $x^{0}$, and $T$ as $S^{0}=\left\{x^{0} ; x \in S\right\}$. Then in $S^{0}$ we have $\left(x^{0}\right)^{-1}=x^{00}$. If $E\left(S^{0}\right)$ is the semilattice of idempotents of $S^{0}$ then the inverse transversal $S^{0}$ is said to be multiplicative if $x^{0} x y y^{0} \in E\left(S^{0}\right)$ for all $x, y \in S[2]$; weakly multiplicative if $\left(x^{0} x y y^{0}\right)^{0} \in E\left(S^{0}\right)$ for all $x, y \in S$ [9]; and a quasi-ideal if $S^{0} S S^{0} \subseteq S^{0}$ [7]. The complicated structure of regular semigroups having inverse transversals of these types has been determined by Saito [10]. Here we shall make use of the following important facts:

(a) $S$ is orthodox if and only if $(x y)^{0}=y^{0} x^{0}$ for all $x, y \in S$, in which case $e^{0} \in E$ for every $e \in E$;

$(\beta)$ if $S$ is orthodox then all inverse transversals are weakly multiplicative;

$(\gamma)(\forall x, y \in S)\left(x y^{0}\right)^{0}=y^{00} x^{0}$ and $\left(y^{0} x\right)^{0}=x^{0} y^{00}$.

Recently, we have shown [5] that an inverse transversal is multiplicative if and only if it is both weakly multiplicative and a quasi-ideal.

Quite often, arguments that concern inverse transversals deal with rather complicated computations involving elements of the form $x^{00}$ and naturally occurring identities such as, for example, $x^{0} x^{00} x^{0}=x^{0} x x^{0}$. Here we consider inverse transversals with respect to which certain words can be simplified similarly.

Definition. Let $S$ be a regular semigroup with an inverse transversal $S^{0}$. Define a balanced word over $S$ to be an element of the form $w=x a_{1} \ldots a_{n} x$, i.e. that begins and

*NATO Collaborative Research Grant 910765 is gratefully acknowledged. 
ends with the same letter $x$ of $S$. Define an inflation of $w$ to be a word obtained from $w$ by replacing any intermediate letter $y_{i}$ by $y_{i}^{00}$, and a deflation of $w$ to be a word obtained from $w$ by replacing any intermediate letter of the form $y_{i}^{00}$ by $y_{i}$. Then we shall say that the inverse transversal is strongly simplistic if every balanced word is invariant under all inflations and deflations.

Thus, for example, relative to a strongly simplistic inverse transversal we have

$$
x a b x=x a^{00} b x=x a b^{00} x=x a^{00} b^{00} x=x(a b)^{00} x .
$$

That this is quite a strong condition is reflected in the following characterisation.

Theorem 1. Let $S$ be a regular semigroup with an inverse transversal $S^{0}$. Then the following statements are equivalent:

(1) $S^{0}$ is strongly simplistic;

(2) $S$ is orthodox and $S^{0}$ is multiplicative.

Proof. $(1) \Rightarrow(2)$ : if (1) holds then, for all $x, y \in S$ we have, on the one hand, since idempotents in $S^{0}$ commute,

$$
y^{0} x^{0} x y y^{0} x^{0}=y^{0} x^{0} x^{00} y^{00} y^{0} x^{0}=y^{0} y^{00} y^{0} x^{0} x^{00} x^{0}=y^{0} x^{0}
$$

and, on the other,

$$
\begin{aligned}
x y y^{0} x^{0} x y & =x y(x y)^{0} x y \cdot y^{0} x^{0} \cdot x y(x y)^{0} x y \\
& =x y(x y)^{0} x^{00} y^{00} y^{0} x^{0} x^{00} y^{00}(x y)^{0} x y \\
& =x y(x y)^{0} x^{00} x^{0} x^{00} y^{00} y^{0} y^{00}(x y)^{0} x y \\
& =x y(x y)^{0} x^{00} y^{00}(x y)^{0} x y \\
& =x y(x y)^{0} x y(x y)^{0} x y \\
& =x y .
\end{aligned}
$$

It follows that $y^{0} x^{0} \in V(x y) \cap S^{0}$ and consequently $y^{0} x^{0}=(x y)^{0}$. Hence $S$ is orthodox.

Now we also have

$$
\begin{aligned}
x y y^{0} x^{0} x y & =x y^{00} y^{0} x^{0} x y \\
& =x y^{00} y^{0} x^{0} x y y^{0} y \\
& =x y^{00} y^{0} x^{0} x^{00} y y^{0} y \\
& =x x^{0} x^{00} y^{00} y^{0} y .
\end{aligned}
$$

It follows that

$$
x y=x x^{0} x^{00} y^{00} y^{0} y
$$

and hence that 


$$
x^{0} x y y^{0}=x^{0} x x^{0} x^{00} y^{00} y^{0} y y^{0}=x^{0} x^{00} y^{00} y^{0} \in E\left(S^{0}\right) .
$$

Consequently, $S^{0}$ is multiplicative.

$(2) \Rightarrow(1)$ : If (2) holds then $S^{0}$ is a quasi-ideal and therefore in particular $x^{0} S x^{0} \subseteq S^{0}$ for every $x \in S$. Consequently, every balanced word $w$ of the form $x^{0} \ldots x^{0}$ belongs to $S^{0}$ and so $w=w^{00}$. Since $S$ is orthodox by hypothesis, we have $(a b)^{00}=a^{00} b^{00}$ for all $a, b \in S$. It follows that every inflation $e$ of $w$ is such that $e=e^{00}=w^{00}=w$, and similarly for every deflation of $w$. Hence all balanced words of the form $x^{0} \ldots x^{0}$ are invariant under inflation or deflation. Now let $y=x \ldots x$ be an arbitrary balanced word. Let $t$ be an inflation of $y$. Then clearly $x x^{0} t=t=t x^{0} x$. Now $x^{0} t x^{0}$ is an inflation of $x^{0} y x^{0}$ so, by the above observation, $x^{0} t x^{0}=x^{0} y x^{0}$. Consequently.

$$
y=x x^{0} y x^{0} x=x x^{0} t x^{0} x=t
$$

Similarly we can show that if $s$ is a deflation of $y$ then $s=y$. We conclude that $S^{0}$ is strongly simplistic.

The characterisation in Theorem 1 shows that the condition that $S^{0}$ be strongly simplistic is quite restrictive. It is natural, therefore, to consider weaker conditions. For this purpose we require the fact, established by Tang [11], that if $E(S)$ is the set of idempotents of $S$ then

$$
\mathbf{I}=\left\{x x^{0} ; x \in S\right\}=\left\{e \in E(S) ; e=e e^{0}\right\}
$$

is a sub-band of $S$; moreover, it is left regular [i.e. $(\forall i, j \in I) i j i=i j]$. Dually,

$$
\Lambda=\left\{x^{0} x ; x \in S\right\}=\left\{f \in E(S) ; f=f^{0} f\right\}
$$

is a sub-band of $S$; moreover, it is right regular [i.e. $(\forall e, f \in \Lambda) e f e=f e$ ].

Observe that In $\Lambda=E\left(S^{0}\right)$, In fact, if $e \in I \cap \Lambda$ then $e=e e^{0}=e^{0} e$ and therefore $e^{0}=e^{0} e e^{0}=e e^{0}=e$, so $e \in E\left(S^{0}\right)$. Conversely, if $e \in E\left(S^{0}\right)$ then $e \in V(e) \cap S^{0}$ gives $e=e^{0}$ and consequently $e \in \mathbf{I} \cap \boldsymbol{\Lambda}$.

Clearly, if $i \in I$ then $i^{0}=i^{0} i i^{0}=i^{0} i$ whence $i^{0} \in E\left(S^{0}\right)$ so that $i^{0} \in V\left(i^{0}\right) \cap S^{0}$ and therefore $i^{0}=i^{00}$. Similarly, if $f \in \Lambda$ then $f^{00}=f^{0} \in E\left(S^{0}\right)$. It follows that the bands $I$ and $\Lambda$ have as inverse transversal the semilattice $E\left(S^{0}\right)$.

Definition. Let $S$ be a regular semigroup with an inverse transversal $S^{0}$. Then we shall say that $S^{0}$ is left simplistic if

$$
(\forall x, y \in S) \quad x^{0} y x^{0}=x^{0} y^{00} y^{0} y x^{0} ;
$$

right simplistic if

$$
(\forall x, y \in S) \quad x^{0} y x^{0}=x^{0} y y^{0} y^{00} x^{0}
$$


and simplistic if it is both left simplistic and right simplistic.

Theorem 2. Let $S$ be a regular semigroup with an inverse transversal $S^{0}$. Then the following statements are equivalent:

(1) $S^{0}$ is left simplistic;

(2) $(\forall i, j \in \mathbf{I}) \quad i j=i j^{0}$;

(3) I is left normal;

(4) I is normal;

(5) $E\left(S^{0}\right)$ is a right ideal of $\mathbf{I}$;

(6) $(\forall x, y, z \in S) \quad x^{0} y z^{0}=x^{0} y^{00} y^{0} y z^{0}$;

(7) $S^{0} \mathbf{I} S^{0} \subseteq S^{0}$;

(8) $\left(\forall x \in S^{0}\right)(\forall i \in I) \quad x i x^{0} \in E\left(S^{0}\right)$;

(9) $(\forall x \in S)(\forall i, j \in I) \quad x i(x i)^{0}$ and $x j(x j)^{0}$ commute.

Proof. $(1) \Rightarrow(2)$ : Suppose that (1) holds. Then for $i, j, k \in \mathbf{I}$ we have

$$
\begin{aligned}
& i^{0} j=i^{0} i j=i^{0} i j i=i^{0} j i=i^{0} j i i^{0} \\
& =i^{0}(j i)^{00}(j i)^{0} j i i^{0} \text { by (1) } \\
& =i^{0} j^{00} i^{00} i^{0} j^{0} j i i^{0} \quad \text { since } I \text { is orthodox } \\
& =i^{0} j^{0} i^{0} i^{0} j^{0} j i i^{0} \\
& =i^{0} j^{0} \quad \text { since } i^{0}, j^{0} \text { commute. }
\end{aligned}
$$

Consequently, $i j=i i^{0} j=i i^{0} j^{0}=i j^{0}$.

(2) $\Rightarrow$ (3): If (2) holds then, for all $i, j, k \in \mathbf{I}$,

$$
i j k=i j k^{0}=i j^{0} k^{0}=i k^{0} j^{0}=i k j^{0}=i k j
$$

Hence $I$ is left normal.

$(3) \Rightarrow(4)$ : This is clear.

$(4) \Rightarrow(5)$ : If (3) holds then for every $x^{0} \in E\left(S^{0}\right)$ and every $i \in I$ we have

$$
\begin{aligned}
x^{0} i=x^{0} i i^{0} i & =x^{0} i^{0} i i & & \text { by }(4) \\
& =x^{0} i^{0} \in E\left(S^{0}\right) & & \text { since } i^{0} \in E\left(S^{0}\right) .
\end{aligned}
$$

Hence $E\left(S^{0}\right) I \subseteq E\left(S^{0}\right)$ and so $E\left(S^{0}\right)$ is a right ideal of $\mathbf{I}$.

$(5) \Rightarrow(6)$ : Since I is orthodox we have, for all $x, y \in S$,

$$
\left(x^{00} x^{0} y y^{0}\right)^{00}=\left(x^{00} x^{0}\right)^{00}\left(y y^{0}\right)^{00}=x^{00} x^{0} y^{00} y^{0} .
$$


Consequently, if (5) holds then, for all $x, y, z \in S$,

$$
\begin{aligned}
x^{0} y z^{0} & =x^{0} x^{00} x^{0} y y^{0} y z^{0} \\
& =x^{0}\left(x^{00} x^{0} y y^{0}\right)^{00} y z^{0} \quad \text { by }(5) \\
& =x^{0} x^{00} x^{0} y^{00} y^{0} y z^{0} \\
& =x^{0} y^{00} y^{0} y z^{0} .
\end{aligned}
$$

(6) $\Rightarrow$ (7): If (6) holds, take $y \in \mathbf{I}$. Then $y=y y^{0}$ and (7) follows.

$(7) \Rightarrow(8)$ : If (7) holds then for $x \in S^{0}$ and $i \in \mathbf{I}$ we have, using $(\gamma), x i x^{0}=\left(x i x^{0}\right)^{00}=x i^{0} x^{0}$ which clearly belongs to $E\left(S^{0}\right)$.

(8) $\Rightarrow$ (9): If (8) holds then for all $x \in S$ and all $i, j \in I$ we have, since I is left regular,

$$
\begin{aligned}
x i(x i)^{0} x j(x j)^{0} & \left.=x x^{0} \cdot x i(x i)^{0} x j(x j)\right)^{0} \cdot x x^{0} x^{00} x^{0} \\
& =x x^{0} \cdot x i(x i)^{0} x j(x j)^{0} \cdot x^{00} x^{0} \\
& =x x^{0}\left(x i(x i)^{0} x j(x j)^{0}\right)^{0} x^{00} x^{0} \text { by }(8) \\
& =x x^{0}\left(x j(x j)^{0} x i(x i)^{0}\right)^{0} x^{00} x^{0} \text { since }(\forall i, j \in \mathbf{I})(i j)^{0}=(j i)^{0} \\
& =x j(x j)^{0} x i(x i)^{0} .
\end{aligned}
$$

(9) $\Rightarrow(3)$ : If (9) holds, choose $x \in k \in \mathbf{I}$. Since then $k i(k i)^{0} k j(k j)^{0}=k i k j=k i j$ it follows from (9) that $k i j=k j i$, so that (3) holds.

$(6) \Rightarrow(1)$ : This is clear.

There is of course a result that is dual to Theorem 2 and gives a characterisation of right simplistic inverse transversals. Combining these, we obtain:

Theorem 3. Let $S$ be a regular semigroup with an inverse transversal $S^{0}$. Then the following statements are equivalent:

(1) $S^{0}$ is simplistic;

(2) $\mathbf{I}$ is (left) normal and $\mathbf{\Lambda}$ is (right) normal;

(3) $E\left(S^{0}\right)$ is a right ideal of $\mathbf{I}$ and a left ideal of $\Lambda$;

(4) $(\forall x, y, z \in S) \quad x^{0} y z^{0}=x^{0} y^{00} z^{0}$;

(5) $(\forall x, y \in S) \quad x^{0} y x^{0}=x^{0} y^{00} x^{0}$;

(6) $S^{0}$ is a quasi-ideal of $S$.

Proof. It is clear from Theorem 2 and its dual that the statements (1), (2), (3) are equivalent.

(3) $\Rightarrow(4)$ : If (3) holds then for all $x, y, z \in S$ we have 


$$
\begin{aligned}
& x^{0} y z^{0}=x^{0} x^{00} x^{0} y y^{0} y z^{0} z^{00} z^{0} \\
& =x^{0} x^{00} x^{0} y^{00} y^{0} y z^{0} z^{00} z^{0} \quad\left[E\left(S^{0}\right) \text { a right ideal of } I\right] \\
& =x^{0} x^{00} x^{0} y^{00} y^{0} y^{00} z^{0} z^{00} z^{0} \quad\left[E\left(S^{0}\right) \text { a left ideal of } \Lambda\right] \\
& =x^{0} y^{00} z^{0} \text {. }
\end{aligned}
$$

(4) $\Rightarrow(5)$ : This is trivial.

(4) $\Rightarrow(6)$ : Clearly, if (4) holds then $S^{0} S S^{0} \subseteq S^{0}$.

$(5) \Rightarrow(1)$ : If (5) holds then for all $x, y \in S$ we have

$$
\begin{array}{rlr}
x^{0} y^{00} y^{0} y x^{0} & =x^{0} x^{00} x^{0} y^{00} y^{0} y x^{0} \\
& =x^{0} y^{00} y^{0} x^{00} x^{0} y x^{0} \\
& =x^{0} y^{00} y^{0} x^{00} x^{0} y^{00} x^{0} \quad \text { by (5) } \\
& =x^{0} x^{00} x^{0} y^{00} y^{0} y^{00} x^{0} \\
& =x^{0} y^{00} x^{0} \\
& =x^{0} y x^{0} &
\end{array}
$$

Consequently, $S^{0}$ is left simplistic. Similarly, $S^{0}$ is right simplistic.

$(6) \Rightarrow(2)$ : This is established in [7, Proposition 1.7].

Using the fact, mentioned above, that an inverse transversal is multiplicative if and only if it is both weakly multiplicative and a quasi-ideal, we can order by logical implication the various properties concerning inverse transversals and thereby obtain the following semilattice:

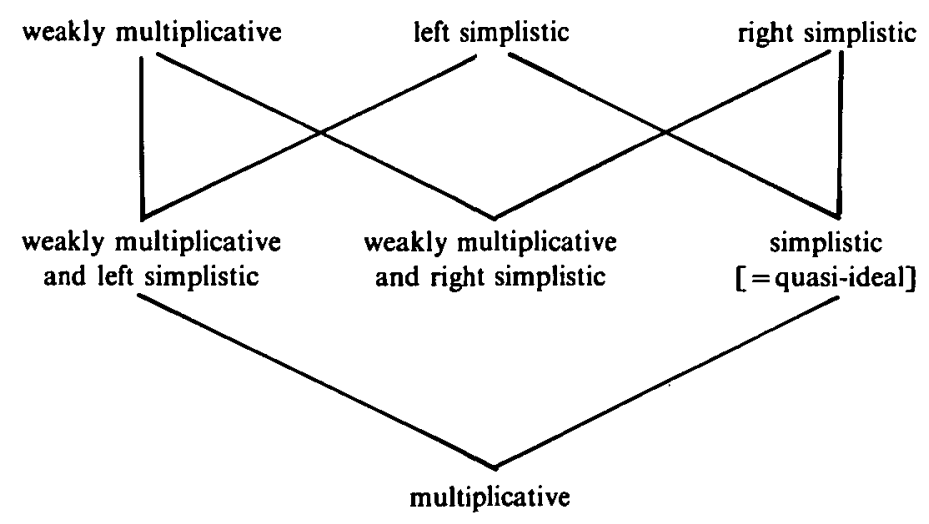

In what follows we shall give a variety of examples, all of which are based on $2 \times 2$ matrices, that describe regular semigroups (orthodox or otherwise, as appropriate) that have the properties described above. We list these examples according to the strongest 
condition satisfied by the inverse transversal $S^{0}$. In some of these we make use of the semigroup Sing $2 \times 2 \mathbb{R}$ of singular real $2 \times 2$ matrices. We let $\operatorname{Sing}_{2 \times 2}^{*} \mathbb{R}$ be the subset of those matrices whose leading element (i.e. that in the $(1,1)$-position) is non-zero.

(a) $S^{0}$ multiplicative.

Here examples are well known. For instance, if $S$ is a naturally ordered regular semigroup with a biggest idempotent $\alpha$ then $\alpha S \alpha$ is a multiplicative inverse transversal of $S[1,3]$. For our purposes below, we provide the following examples of terms of $2 \times 2$ matrices.

Example 1 [orthodox]. Let $S_{1}$ be the subset of $\operatorname{Sing}_{2 \times 2}^{*} \mathbb{R}$ described by

$$
S_{1}=\left\{\left[\begin{array}{ll}
x & y \\
0 & 0
\end{array}\right] ; \quad x, y \in \mathbb{R}, \quad x \neq 0\right\}
$$

Then $S_{1}$ is a semigroup. It is readily seen that $\left[\begin{array}{cc}x^{-1} & 0 \\ 0 & 0\end{array}\right] \in V\left[\begin{array}{ll}x & y \\ 0 & 0\end{array}\right]$ and so $S_{1}$ is regular. The idempotents are the matrices of the form $\left[\begin{array}{ll}1 & y \\ 0 & 0\end{array}\right]$ and consequently $S_{1}$ is orthodox. If we define $\left[\begin{array}{ll}x & y \\ 0 & 0\end{array}\right]^{0}=\left[\begin{array}{cc}x^{-1} & 0 \\ 0 & 0\end{array}\right]$ then the group $S_{1}^{0}=\left\{\left[\begin{array}{ll}x & y \\ 0 & 0\end{array}\right] ; x \neq 0\right\}$ is such that

$$
V\left[\begin{array}{ll}
x & y \\
0 & 0
\end{array}\right] \cap S_{1}^{0}=\left\{\left[\begin{array}{cc}
x^{-1} & 0 \\
0 & 0
\end{array}\right]\right\}
$$

and is therefore an inverse transversal of $S_{1}$. Again as can readily be verified, we have

$$
\begin{aligned}
& \mathbf{I}=\left\{X X^{0} ; X \in S_{1}\right\}=\left\{\left[\begin{array}{ll}
1 & 0 \\
0 & 0
\end{array}\right]\right\}=E\left(S_{1}^{0}\right), \\
& \Lambda=\left\{X^{0} X ; X \in S_{1}\right\}=\left\{\left[\begin{array}{ll}
1 & z \\
0 & 0
\end{array}\right] ; z \in \mathbb{R}\right\}=E\left(S_{1}\right)
\end{aligned}
$$

from which it follows that $\mathbf{\Lambda I}=E\left(S_{1}^{0}\right)$ and therefore $S_{1}^{0}$ is multiplicative.

Example 2 [non-orthodox]. Let $F$ be a field of characteristic 2 and consider the subset $S_{2}$ of Mat $_{2 \times 2} F$ given by

$$
S_{2}=\left\{\left[\begin{array}{ll}
1 & 1 \\
1 & 1
\end{array}\right],\left[\begin{array}{ll}
1 & 0 \\
1 & 0
\end{array}\right],\left[\begin{array}{ll}
1 & 1 \\
0 & 0
\end{array}\right],\left[\begin{array}{ll}
1 & 0 \\
0 & 0
\end{array}\right],\left[\begin{array}{ll}
0 & 0 \\
0 & 0
\end{array}\right]\right\} \equiv\{a, b, c, d, e\}
$$


The only non-idempotent of $S_{2}$ is $a$, and clearly $d \in V(a)$. Hence $S_{2}$ is regular. That $S_{2}$ is not orthodox follows from the equality $b c=a$. It is readily verified that

$$
V(a)=\{d\}, \quad V(b)=\{d, b\}, \quad V(c)=\{d, c\}, \quad V(d)=\{d, a, b, c\}, \quad V(e)=\{e\} .
$$

Defining

$$
a^{0}=b^{0}=c^{0}=d^{0}=d, \quad e^{0}=e,
$$

we therefore obtain an inverse (in fact, semilattice) transversal $S_{2}^{0}=(d, e\}$.

Simple calculations reveal that $\mathbf{I}=\{b, d, e\}$ and $\boldsymbol{\Lambda}=\{c, d, e\}$. It follows that $\mathbf{\Lambda I}=\{d, e\}$ and so $S_{2}^{0}$ is multiplicative.

(b) $S^{0}$ weakly multiplicative and left simplistic.

Example 3 [orthodox]. Let $S_{3}$ be the semigroup that consists of $S_{1}$ with the $2 \times 2$ identity matrix adjoined. Then, as shown in [4], $S_{3}$ is right inverse. Moreover,

$$
\left[\begin{array}{cc}
x^{-1} & x^{-1} \\
0 & 0
\end{array}\right] \in V\left[\begin{array}{cc}
x & y \\
0 & 0
\end{array}\right],
$$

and consequently

$$
S_{3}^{0} \equiv\left\{\left[\begin{array}{ll}
x & x \\
0 & 0
\end{array}\right] ; x \neq 0\right\} \cup\left\{\left[\begin{array}{ll}
1 & 0 \\
0 & 1
\end{array}\right]\right\}
$$

is an inverse transversal of $S_{3}$ which is weakly multiplicative but not multiplicative. Since $S_{3}$ is right inverse, it satisfies the identity $x=x^{00} x^{0} x$ and consequently $S_{3}^{0}$ is left simplistic. That $S_{3}^{0}$ is not right simplistic follows from the fact that $\Lambda=E\left(S_{3}\right)$ and does not contain $E\left(S_{3}^{0}\right)$ as a left ideal.

Example 4 [non-orthodox]. The semigroup $S_{4}=S_{2} \times S_{3}$, where $S_{2}$ is an Example 2 and $S_{3}$ is as in Example 3 , suffices. The inverse transversal in question is $S_{2}^{0} \times S_{3}^{0}$. Equivalently, we can consider the semigroup of $4 \times 4$ matrices of the form

$$
\left[\begin{array}{ll}
A & 0 \\
0 & B
\end{array}\right]
$$

where $A \in S_{2}, B \in S_{3}$, and all other entries are 0 .

(c) $S^{0}$ weakly multiplicative and right simplistic.

Example 5 [orthodox]. Simply form $S_{5}$ from $S_{3}$ above by taking the transposes of all the matrices. 
Example 6 [non-orthodox]. Take $S_{6}=S_{2} \times S_{5}$.

(d) $S^{0}$ weakly multiplicative.

Example 7 [orthodox]. Take $S_{7}=S_{3} \times S_{5}$. Equivalently, consider the semigroup of $4 \times 4$ matrices of the form

$$
\left[\begin{array}{ll}
A & 0 \\
0 & B
\end{array}\right]
$$

where $A \in S_{3}, B \in S_{5}$, and all other entries are 0 .

Example 8 [non-orthodox]. In [5] we have shown that if $B$ is a boolean algebra then the semigroup $B_{2}=\operatorname{Mat}_{2 \times 2} B$ is regular and non-orthodox. Moreover, every $\left[\begin{array}{ll}a & b \\ c & d\end{array}\right] \in B_{2}$ has a biggest inverse, namely

$$
\left[\begin{array}{ll}
a & b \\
c & d
\end{array}\right]^{0}=\left[\begin{array}{ll}
b^{\prime}(a+c)+c^{\prime}(a+b)+d & a^{\prime}(c+d)+b^{\prime}(a+c)+b \\
a^{\prime}(b+d)+b^{\prime}(a+d)+c & b^{\prime}(c+d)+c^{\prime}(b+d)+a
\end{array}\right]
$$

and the set $B_{2}^{0}$ so described is an inverse transversal of $B_{2}$ that is weakly multiplicative but not multiplicative. From the descriptions of $E\left(B_{2}^{0}\right)$ and of $I$ given in [5] we have in particular that if $X=\left[\begin{array}{ll}a & a \\ 0 & 0\end{array}\right]$ then $X \in I$ and $X \notin B_{2}^{\circ}$. Consequently, if $E\left(B_{2}^{0}\right)$ were a right ideal of 1 then we would have the contradiction $X=I_{2} X \in E\left(B_{2}^{0}\right)$. Hence $B_{2}^{0}$ is not left simplistic; and similarly it is not right simplistic.

(e) $S^{0}$ a quasi-ideal.

Here examples are again well known and include rectangular bands of inverse semigroups [8] and four-spiral semigroups [6]. Note that, as observed above, when $S$ is orthodox all inverse transversals are weakly multiplicative. In what follows, therefore, all examples have to be non-orthodox. The following matrix example will be useful in another context.

Example 9. Observe that Sing $g_{2 \times 2}^{*} \mathbb{R}$ consists of matrices of the form

$$
\left[\begin{array}{cc}
a & b \\
c & a^{-1} b c
\end{array}\right]
$$

where $a, b, c \in \mathbb{R}$ with $a \neq 0$. Let $M$ be the set $\operatorname{Sing}_{2 \times 2}^{*} \mathbb{R}$ with the $2 \times 2$ zero matrix adjoined. Then $M$ is a semigroup. This can be verified by considering the product 


$$
\left[\begin{array}{cc}
a & b \\
c & a^{-1} b c
\end{array}\right]\left[\begin{array}{cc}
x & y \\
z & x^{-1} y z
\end{array}\right]=\left[\begin{array}{cc}
a x+b z & a y+b x^{-1} y z \\
c x+a^{-1} b c z & c y+a^{-1} b c x^{-1} y z
\end{array}\right]
$$

and observing that if $a x+b z=0$ then every element of the matrix on the right hand side is 0 . That $M$ is regular follows, for example, from the fact that if $a \neq 0$ then, by a simple calculation,

$$
\left[\begin{array}{cc}
a^{-1} & 0 \\
0 & 0
\end{array}\right] \in V\left[\begin{array}{cc}
a & b \\
c & a^{-1} b c
\end{array}\right]
$$

That $M$ is not orthodox can easily be verified. For example, each of the matrices

$$
A=\left[\begin{array}{ll}
2 & -2 \\
1 & -1
\end{array}\right], \quad B=\left[\begin{array}{rr}
-1 & -2 \\
1 & 2
\end{array}\right]
$$

belongs to $M$ and is idempotent but $A B$ is not idempotent.

Consider now the subset

$$
M^{0}=\left\{\left[\begin{array}{ll}
x & 0 \\
0 & 0
\end{array}\right] ; x \neq 0\right\} \cup\left\{\left[\begin{array}{ll}
0 & 0 \\
0 & 0
\end{array}\right]\right\}
$$

which, being clearly a group with a zero element adjoined, is an inverse subsemigroup of $M$. Since

$$
M^{0} \cap V\left[\begin{array}{cc}
a & b \\
c & a^{-1} b c
\end{array}\right]=\left\{\left[\begin{array}{cc}
a^{-1} & 0 \\
0 & 0
\end{array}\right]\right\}, \quad M^{0} \cap V\left[\begin{array}{ll}
0 & 0 \\
0 & 0
\end{array}\right]=\left\{\left[\begin{array}{ll}
0 & 0 \\
0 & 0
\end{array}\right]\right\}
$$

we have that $M^{0}$ is an inverse transversal of $M$, so we can define appropriately

$$
\left[\begin{array}{cc}
a & b \\
c & a^{-1} b c
\end{array}\right]^{0}=\left[\begin{array}{cc}
a^{-1} & 0 \\
0 & 0
\end{array}\right],\left[\begin{array}{ll}
0 & 0 \\
0 & 0
\end{array}\right]^{0}=\left[\begin{array}{ll}
0 & 0 \\
0 & 0
\end{array}\right]
$$

Further easy calculations show that

$$
\begin{aligned}
& I=\left\{X X^{0} ; X \in M\right\}=\left\{\left[\begin{array}{ll}
1 & 0 \\
x & 0
\end{array}\right] ; x \in \mathbb{R}\right\} \cup\left\{\left[\begin{array}{ll}
0 & 0 \\
0 & 0
\end{array}\right]\right\}, \\
& \Lambda=\left\{X^{0} X ; X \in M\right\}=\left\{\left[\begin{array}{ll}
1 & x \\
0 & 0
\end{array}\right] ; x \in \mathbb{R}\right\} \cup\left\{\left[\begin{array}{ll}
0 & 0 \\
0 & 0
\end{array}\right]\right\} .
\end{aligned}
$$

Consider now the semilattice 


$$
E\left(M^{0}\right)=\left\{\left[\begin{array}{ll}
1 & 0 \\
0 & 0
\end{array}\right], \quad\left[\begin{array}{ll}
0 & 0 \\
0 & 0
\end{array}\right]\right\}
$$

Clearly, $E\left(M^{0}\right)$ is a right ideal of $\mathbf{I}$ and a left ideal of $\boldsymbol{\Lambda}$. It follows by Theorem 3 that the inverse transversal $M^{0}$ is a quasi-ideal of $M$. Since a typical non-zero element of $\Lambda \mathbf{I}$ is

$$
\left[\begin{array}{ll}
1 & x \\
0 & 0
\end{array}\right]\left[\begin{array}{ll}
1 & 0 \\
y & 0
\end{array}\right]=\left[\begin{array}{cc}
1+x y & 0 \\
0 & 0
\end{array}\right]
$$

and since this does not in general belong to $E\left(M^{0}\right)$ we see that $M^{0}$ is not multiplicative.

(f) $S^{0}$ left simplistic.

Example 10. The semigroup $M \times S_{3}$, where $M$ is as in Example 9 and $S_{3}$ is as in Example 3, suffices. Equivalently, we can consider the semigroup of $4 \times 4$ matrices of the form

$$
\left[\begin{array}{ll}
A & 0 \\
0 & B
\end{array}\right]
$$

where $A \in M, B \in S_{3}$, and all other entries are 0 .

(g) $S^{0}$ right simplistic.

Example 11. Take $M \times S_{5}$, or transposes of the matrices in Example 10 .

It is natural to complete this list of examples by illustrating a regular semigroup $S$ with an inverse transversal $S^{0}$ that does not satisfy any of the above properties.

Example 12. Consider the semigroup $M$ of Example 9. If $M^{1}=M \cup\left\{I_{2}\right\}$ then an inverse transversal of $M^{1}$ is $\left(M^{1}\right)^{0}=\left(M^{0}\right)^{1}$. Moreover, $E\left(M^{1}\right)=E(M)^{1}$ and the corresponding bands are $\mathbf{I}^{1}$ and $\boldsymbol{\Lambda}^{1}$. Now

$$
\mathbf{I}^{1}=I_{2} \mathbf{I}^{1} \subseteq E\left(\left(M^{1}\right)^{0}\right) \mathbf{I}^{1} \subseteq \mathbf{I}^{1} \mathbf{I}^{1}=\mathbf{I}^{1}
$$

and so $E\left(\left(M^{1}\right)^{0}\right) I^{1}=I^{1}$. Since $E\left(\left(M^{1}\right)^{0}\right) \subset I^{1}$ it follows that $E\left(\left(M^{1}\right)^{0}\right)$ is not a right ideal of $I^{1}$. Similarly, it is not a left ideal of $\Lambda^{1}$. Hence $\left(M^{1}\right)^{0}$ is neither left simplistic nor right simplistic. Now $M^{0}$ is not weakly multiplicative. Indeed, if it were, then since it is a quasi-ideal it would be multiplicative; and as shown in Example 9 this is not the case. Consequently, $\left(M^{1}\right)^{0}$ is not weakly multiplicative. These observations show that the inverse transversal $\left(M^{1}\right)^{0}$ satisfies none of the properties considered above. 
As Example 12 illustrates, the situation is quite different in the presence of an identity element. In relation to this, more can be said: if $S$ is a monoid and $S^{0}$ is left simplistic or right simplistic then $S$ is necessarily orthodox. This follows from the following result and its dual.

Theorem 4. Let $S$ be a regular monoid with an inverse transversal $S^{0}$. Then the following statements are equivalent:

(1) $S^{0}$ is left simplistic;

(2) $S$ is right inverse.

Proof. $(1) \Rightarrow(2)$ : If (1) holds then since $1 \in E\left(S^{0}\right)$ we have, by Theorem 3 ,

$$
\mathbf{I}=\mathbf{I} \mathbf{I} \subseteq E\left(S^{0}\right) \mathbf{I} \subseteq E\left(S^{0}\right)
$$

and therefore $\mathbf{I}=E\left(S^{0}\right)$. It follows that

$$
\mathbf{\Lambda} \mathbf{I}=\mathbf{\Lambda} E\left(S^{0}\right) \subseteq \Lambda \mathbf{\Lambda}=\mathbf{\Lambda}
$$

and therefore $(\Lambda I)^{0} \subseteq \Lambda^{0} \subseteq I=E\left(S^{0}\right)$. Consequently, $S^{0}$ is weakly multiplicative. It follows by [9] that if $\langle E(S)\rangle$ is the subsemigroup generated by the idempotents then

$$
\langle E(S)\rangle=\mathbf{I} \mathbf{\Lambda}=E\left(S^{0}\right) \mathbf{\Lambda} \subseteq \mathbf{\Lambda} \mathbf{\Lambda}=\mathbf{\Lambda} \subseteq\langle E(S)\rangle .
$$

Thus we see that $\langle E(S)\rangle=\Lambda$, from which it follows that $S$ is orthodox with $E(S)=\Lambda$. Consequently, every idempotent of $S$ is of the form $x^{0} x$ and therefore Green's relation $\mathscr{L}$ reduces to equality on $E(S)$. In other words, $S$ is right inverse.

$(2) \Rightarrow(1)$ : If (2) holds then, by [4], the identity $x=x^{00} x^{0} x$ holds in $S$ and therefore $S^{0}$ is left simplistic.

Our final example shows that when $S^{0}$ is multiplicative the presence of an identity element is less dramatic. by

Example 13. Let $B$ be a boolean algebra and consider the subsets of $\mathrm{Mat}_{2 \times 2} B$ given

$$
X=\left\{\left[\begin{array}{cc}
x & 0 \\
0 & 0
\end{array}\right] ; x \in B\right\}, \quad Y=\left\{\left[\begin{array}{cc}
x & 0 \\
x & 0
\end{array}\right] ; x \in B\right\}
$$

Under matrix multiplication, the set $Z=X \cup Y$ is a band in which

$$
V\left[\begin{array}{ll}
x & 0 \\
0 & 0
\end{array}\right]=\left\{\left[\begin{array}{ll}
x & 0 \\
0 & 0
\end{array}\right], \quad\left[\begin{array}{ll}
x & 0 \\
x & 0
\end{array}\right]\right\}=V\left[\begin{array}{ll}
x & 0 \\
x & 0
\end{array}\right]
$$

Consequently, defining 


$$
\left[\begin{array}{ll}
x & 0 \\
0 & 0
\end{array}\right]^{0}=\left[\begin{array}{ll}
x & 0 \\
x & 0
\end{array}\right]^{0}=\left[\begin{array}{ll}
x & 0 \\
x & 0
\end{array}\right]
$$

we have that $Z^{0}=Y$ is a semilattice transversal of $Z$. It is readily seen that $I=Z$ and $\Lambda=Y$, so that

$$
\mathbf{\Lambda} \mathbf{I}=Y Z=Y=Z^{0}=E\left(Z^{0}\right)
$$

and therefore $Z^{0}$ is multiplicative.

Consider now the band $Z^{1}=Z \cup\left\{I_{2}\right\}$. The semilattice transversal $\left(Z^{1}\right)^{0}=\left(Z^{0}\right)^{1}$ is then weakly multiplicative but not multiplicative, for if $A=\left[\begin{array}{ll}x & 0 \\ 0 & 0\end{array}\right]$ with $x \neq 0$ then

$$
I_{2}^{0} I_{2} A A^{0}=A \notin Y=E\left(Z^{0}\right)
$$

Since, as is readily verified, the identity $x x^{0} x^{00}=x$ is satisfied in $Z^{1}$, it follows that $\left(Z^{1}\right)^{0}$ is right simplistic.

\section{REFERENCES}

1. T. S. BLYth and R. MCFADDEN, Naturally ordered regular semigroups with a greatest idempotent, Proc. Roy. Soc. Edinburgh 91A (1981), 107-122.

2. T. S. BLYTH and R. MCFADDEN, Regular semigroups with a multiplicative inverse transversal, Proc. Roy. Soc. Edinburgh 92A (1982), 253-270.

3. T. S. Blyth and M. H. Almeida Santos, On naturally ordered regular semigroups with biggest idempotents, Comm. Algebra 21 (1993), 1761-1771.

4. T. S. Blyth and M. H. Almeida Santos, Amenable orders on orthodox semigroups, $J$. Algebra 169 (1994), 49-70.

5. T. S. Blyth and M. H. Almeida Santos, On weakly multiplicative inverse transversals, Proc. Edinburgh Math. Soc. 37 (1994), 91-99.

6. K. Byleen, Regular four-spiral semigroups, idempotent semigroups and the Rees construction, Semigroup Forum 22 (1981), 97-100.

7. D. B. MCAlister and R. McFadden, Regular semigroups with inverse transversals, Quart. $J$. Math. Oxford 34 (1983), 459-474.

8. F. PastiJn, Rectangular bands of inverse semigroups, Simon Stevin 56 (1982), 1-97.

9. Tatsuhiko Saito, Regular semigroups with a weakly multiplicative inverse transversal, in Proc. 8th Symposium on Semigroups (Shimane University, 1985), 22-25.

10. Tatsuhiko Salto, Construction of regular semigroups with inverse transversals, Proc. Edinburgh Math. Soc. 32 (1989), 41-51.

11. XILIN TANG, Regular semigroups with inverse transversals, preprint.

Mathematical Institute

UNIVERSITY OF ST ANDREWS

SCOTLAND
Departamento de Matemática

F.C.T.

Universidade Nova de Lisboa

Portugal 